\title{
Comparison between Covid-19 scenarios in India and other large-population regions
}

\author{
Sudhakar Yarlagadda ${ }^{1,2}$ and Satyaki $\mathrm{Kar}^{3}$ \\ ${ }^{1}$ Academic Council, Visva-Bharati Univ., Santiniketan, West Bengal \\ ${ }^{2}$ Saha Institute of Nuclear Physics, Kolkata, India and \\ 3 Department of Physics, Aghorekamini Prakashchandra \\ Mahavidyalaya, Bengai, West Bengal 712611, India
}

(Dated: April 30, 2020)

\begin{abstract}
We analyze the Covid-19 scenario in India and compare it with those in other large-population regions such as Asia-excluding-China, Africa, European Union, South America, and USA. We compare existing fatality data and offer an interpretation based on inherent immunity and climate in various regions. We also present an India-specific strategy of releasing low vulnerability group from the lockdown and estimate the time required to attain herd immunity. Whatever optimism we present should be viewed as a guarded optimism. There should not be room for complacency.
\end{abstract}

Corresponding author's email: y.sudhakar@saha.ac.in

Keywords: Covid-19, Indian scenario, Inherent immunity, Hot climate 


\section{INTRODUCTION}

The World Health Organization (WHO), on 30/Jan./2020, declared the novel coronavirus outbreak (2019-nCoV) to be a Public Health Emergency of International Concern (PHEIC) ${ }^{1}$. Next, when the virus spread internationally, on 11/March/2020 the WHO declared COVID-19 a "pandemic"213. The scenario regarding the primary driver of transmission is still evolving 4 , and the incubation period ranges between 2 and 14 days 67 . The COVID-19 pandemic presents an urgent challenge to understand and formulate a strategy to deal with infection spreading and fatalities. Rising up to the need, there are some number of sources for data on infections and fatalities for various affected countries/regions in the world 8 - 10 .

There is a large variation in the number of tests-per-million done by various countries to identify COVID-19 patients. Some of the determining factors are the strategies for testing, availability of testing kits, experienced personnel to handle the testing, etc. Especially when the tests are done based on symptoms shown and when the number of asymptomatic cases is several times that of the symptomatic cases (as shown by the COVID-19 data), the number of detected cases will certainly be much smaller than the actual number. Furthermore, initially when there is no lockdown and there is an exponential growth in the number of infected people, the number of tests conducted may not be able to keep in pace with the growing number of infections. Thus it is important to realize that the number of infected cases reported by various countries will not be a good measure of the spread of infection in those countries even for comparative purposes. On the other hand, the number of fatalities is likely to be not in serious error as the detection of death due to severe acute respiratory syndrome is not difficult to diagnose. The main purpose of the present paper is to do a comparative study of the fatalities due to COVID-19 in India and other large-population (i.e., greater than 300 million population) regions such as USA, South America, Africa, European Union, and Asia-excluding-China. Based on the comparative analysis, we aim to offer a strategy of how India should deal with the COVID-19 situation. Part of the motivation for this work stems from the predictions of large-scale deaths in India by groups such as Eili Klein et al!11. 


\section{ANALYSIS}

The following are some points used in our analysis:

1) Counting the number of infected cases for comparison between countries does not make sense as number of cases increases with number of tests. Testing based on symptoms is not indicative of number of infected as ICMR seems to be claiming that $80 \%$ of infected are asymptomatic.

2) Number of deaths is much more a reliable measure. You cannot be wrong by more than a factor of two even if there is a large amount of misdiagnosis/fudging. Anyway, finally what matters is the number of deaths.

3) It is generally believed that, because of various factors (some of which are mentioned below), Indians have immunity protection. Based on information such as the world maps of TB endemic countries and malaria endemic countries (see Figs. 1 and 2), we see that the countries most effected by TB and malaria are, in general, turning out to be least effected by Covid-19 (for Covid-19 information, see Refs. 9 and 10). Thus, one can draw the conclusion that Indians, Africans, etc. have certain immunity.

It is also likely that climate (hot sunny weather and humidity) may help Indians. If hot summer reduces damage, then lockdown in March and April certainly helps.

4) Modeling based on climate and immunity is not easy. However, one can conservatively forecast based on existing trends. Present death rates do indicate that India has lower death rates. Method of comparison is as follows.

We compare India and other large-population (i.e., greater that 300 million population) regions such as Asia-excluding-China, Africa, European Union, South America, and USA. Starting point of comparison is when India crossed 100 deaths and when others hit the number of deaths given by $100 \times$ (population of region/population of India).

From data in https://ourworldindata.org/grapher/total-covid-deaths-region ${ }^{10}$ (also see Fig. 3):

India crossed 100 deaths on Apr./6

USA hit 26 deaths on March/10.

European Union reported 37 deaths on March/2.

South America had 37 deaths on March/22.

On adding 21 days to starting point: 
On Apr./27 deaths in India were 872.

On March/31 deaths in USA were 3170.

On March/23 European Union death count was 8613.

On Apr./12 South America's death tally was 1935.

It is clear that India deaths are less than one-third those of USA, less than one-ninth those of European Union (EU), and less than half those of S. America.

In spite of the fact that people in USA have about 12 times the area-per-person as Indians, people of EU have more than 4 times the area-per-person as Indians, and South American's have more than 16 times the area-per-person as Indians (see Table. I), India seems to be doing much better; we believe this is indicative of immunity based on usual prevalence of malaria and TB. (see Figs. 1 and 2).

Countries with indigenous cases in $\mathbf{2 0 0 0}$ and their status by 2017 Countries with zero indigenous cases over at least the past 3 consecutive years are considered to be malaria free. All countries in the WHO European Region reported zero indigenous cases in 2016 and again in 2017. In 2017, both China and El Salvador reported zero indigenous cases. Source: WHO database.

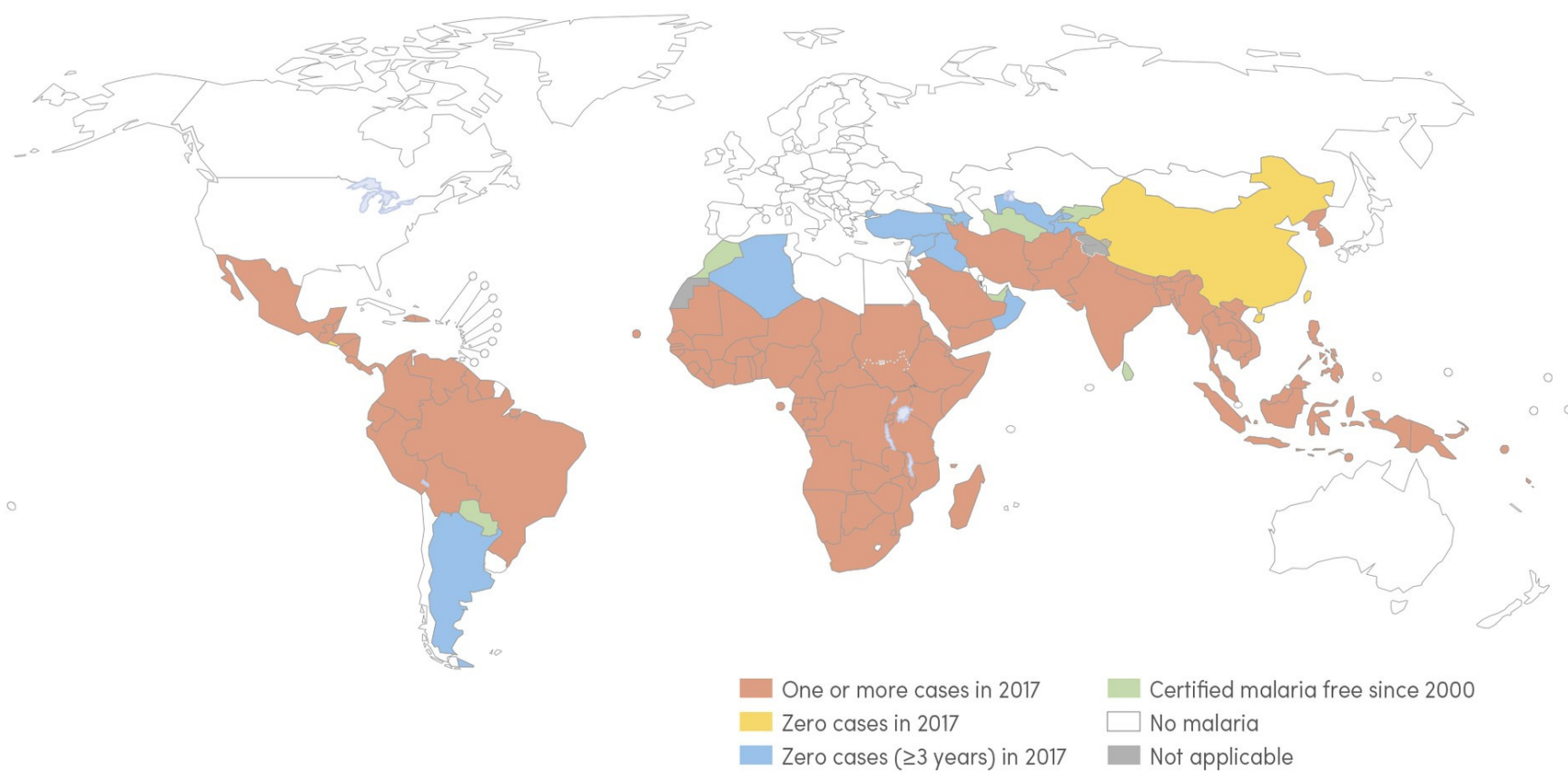

WHO: World Health Organization.

FIG. 1. (Color online) World map of malaria endemic countries 12 . 


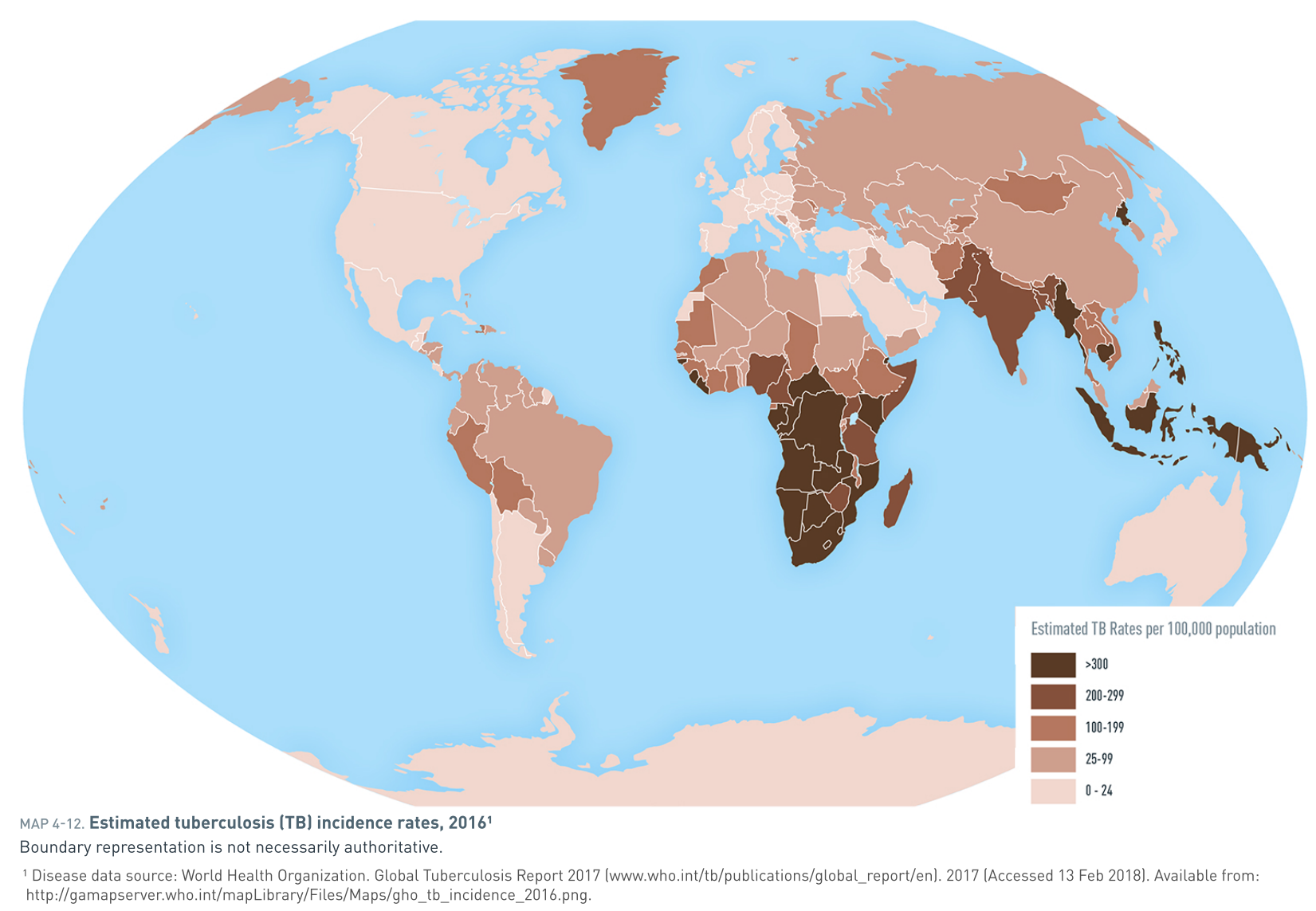

FIG. 2. (Color online) World map of TB endemic countries 13 .

The population of the African continent and India's population are roughly the same (see Table I). Their trajectories for total fatalities (see Fig . 3) are also similar. Since, like Indians, Africans also have immunity, the death rates of these two regions can be comparable. However, since Africa has about 10 times India's area, better Indian response (lockdown, medical treatment, etc.) probably overcame the area disadvantage and helped India keep the death-numbers down. Lastly, Asia-excluding-China, after 21 days from the starting point March/9 (when it reported 261 deaths), had a death tally of 3364 which is clearly more than 2020, i.e., the Indian number modified in proportion to the population $(872 \times 3196 / 1380 \approx 2020)$. Furthermore, in Asia-excluding-China the area per-person is more than 4 times that in India. Perhaps it is a combination of better immunity (see Figs. 1 and 2) and Indian response that helped India fare better.

It is also interesting to compare the best scenario country in Europe (i.e., Germany) with the worst case in Asia (i.e., Iran). Both Iran and Germany have approximately 82 million 


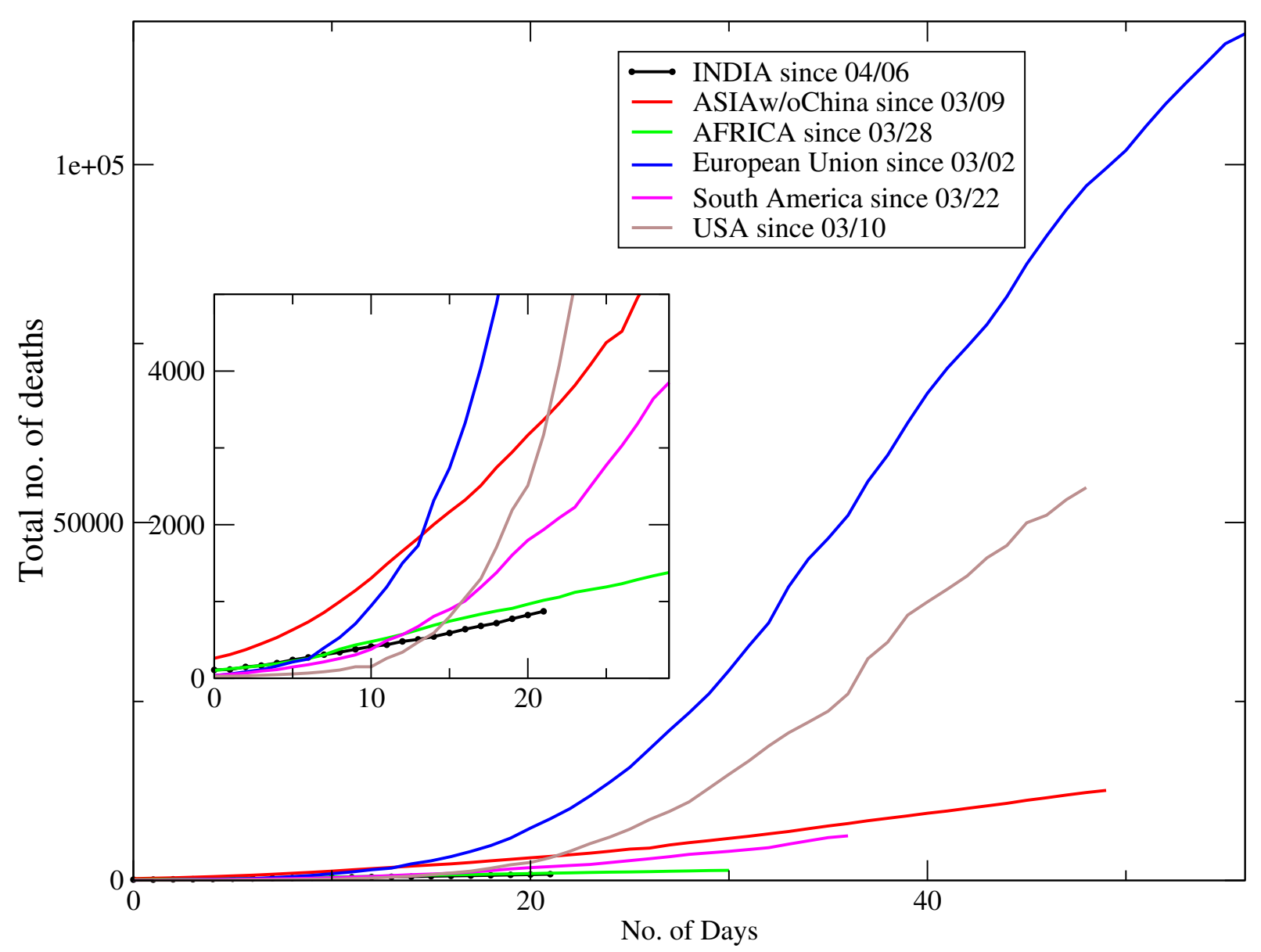

FIG. 3. (Color online) Total fatalities as reported in "our world in data"10. Starting point of comparison is when India reported 100 deaths and when other regions reported the number of deaths given by $100 \times($ population of region/population of India)

people. Iran started being a hotspot before Germany, yet both have reported approximately same number of deaths. More exactly, (from data in https://www.worldometers.info/coronavirus $/{ }^{9}$ ) as on Apr./25, Iran has had 5650 deaths while Germany reported 5877 deaths. In fact, a reason why Iran has higher deaths among Asian countries could be that, although malaria infections exist in Iran, it has low prevalence of TB.

As regards climate, hot sunny weather seems to help keep the death-count low in countries such as Australia, New Zealand, and Argentina which have recently come out of summer in the southern hemisphere; these countries do not have prevalence of malaria or TB. From Fig. 4, it is obvious that the fatality count is low, possibly due to hot sunny climate, even in countries with moderate population such as Argentina and Australia (see Table. I). It should be noted that, in Fig. 4, the starting point for each coun- 


\begin{tabular}{|c|c|c|}
\hline Region & Population (Millions) & Area (Million Sq. Kms.) \\
\hline India & 1380 & 3.29 \\
\hline Asia w/o China & 3196 & 34.98 \\
\hline Africa & 1335 & 30.37 \\
\hline European Union & 446 & 4.48 \\
\hline South America & 430 & 17.84 \\
\hline USA & 331 & 9.83 \\
\hline Argentina & 45.1 & 2.78 \\
\hline Australia & 25.4 & 7.69 \\
\hline NewZealand & 4.8 & 0.27 \\
\hline
\end{tabular}

TABLE I. Comparison of populations and land areas of various regions (based on Wikipedia)

try is when the first death was reported. This is because the number of deaths given by $100 \times($ population of country/population of India) is of the order unity.

\section{CONCLUSIONS AND WAY FORWARD}

- One needs caution extrapolating based on existing trends. As always extrapolation is less predictable than interpolation.

- India needs to have its own strategy regarding when to end the lockdown and develop its own plan of action that keeps its people and economy in mind.

- Given lower fatalities, easing lockdown can be earlier and broader. See below for a possible strategy.

- People most exposed to TB, malaria, etc. are best suited to generate herd immunity.

- India needs to develop its own strategy for generating herd immunity. See below for a possible strategy.

The lockdown can be ended gradually (with continued monitoring of the response) based on age group vulnerability and only for people without medical conditions such as diabetes, 
asthma, heart problems, hypertension, etc. For instance, they can start with the least vulnerable age group of below 40 years (i.e., more than 70\% population based on 2011 census), then include age groups between 40 and 50 years (i.e., more than $11 \%$ of population), and if the response is good maybe include people between 50 and 60 years (i.e., more than $7 \%$ of the population) at a later stage.

Assuming $R_{0}$ (the reproduction number) ${ }^{14}$ is 2.5 , the herd immunity threshold (HIT) is given by $\left(1-1 / R_{0}\right) \times 100$, i.e., $60 \%$ population which is about $1.38 \times 0.60 \approx 0.83$ billion. On releasing only people below 50 years from lockdown, from the corresponding group of about 1.1 billion (i.e., $0.8 \times 1.38$ billion), we calculate the time to attain herd immunity threshold. Assuming that each person takes (on an average) 7 days to infect 2.5 people (on an average) and given that $2.5^{20} \approx 0.9$ billion, one can expect to attain HIT in about 5 months. Alternately, (based on current trends given in Ref. 15] during lockdown) we conservatively assume that growth rate in number of cases is $6 \%$ and that existing infected cases are

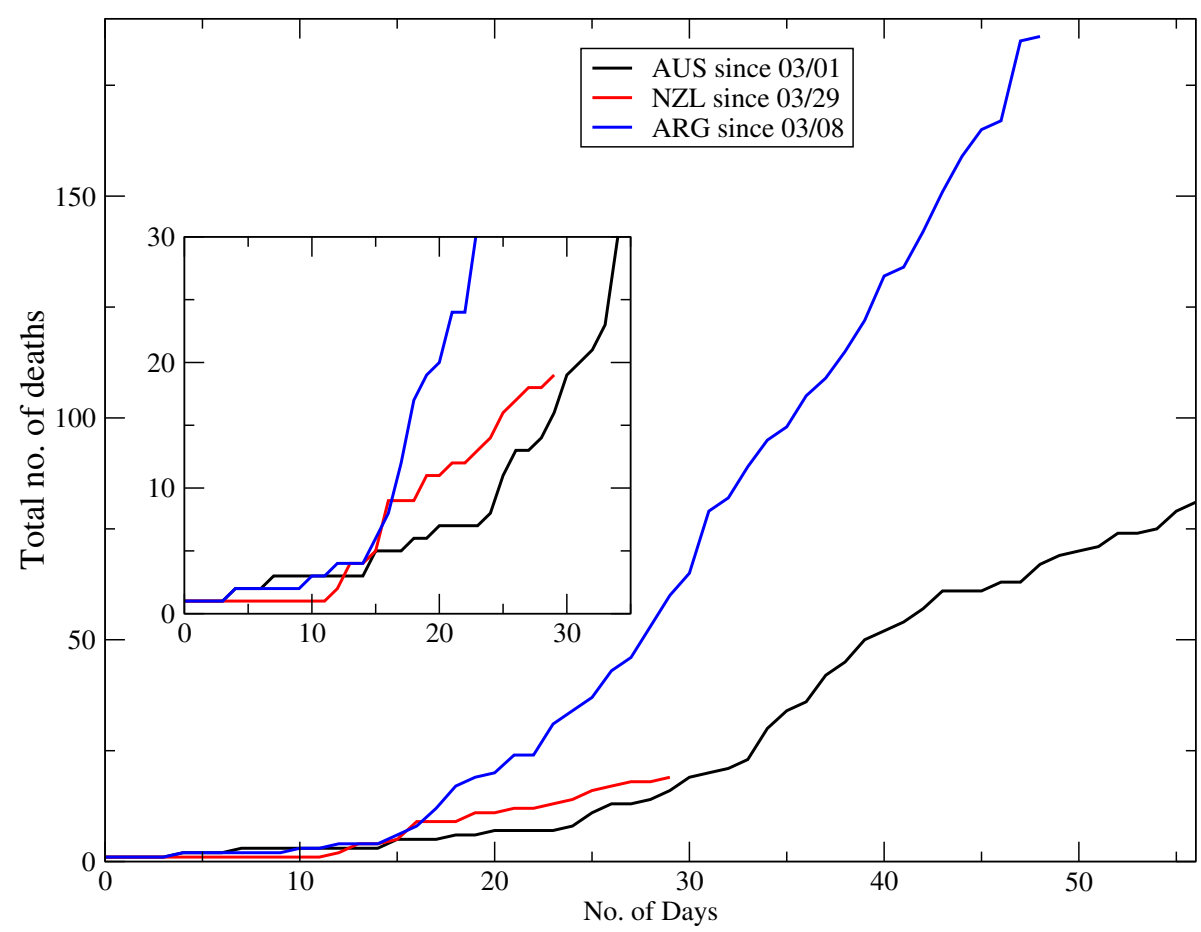

FIG. 4. (Color online) Total fatalities as reported in "our world in data" ${ }^{10}$ for a few countries (Argentina, Australia, and New Zealand) in the southern hemisphere that do not have prevalence of malaria or TB. The starting point for all the countries is when the first death was reported in that country. 
about 1.4 lakhs (with 20000 being detected while remaining 120000 being undetected and asymptomatic); then, after 5 months from now, the number of infected cases is approximately 0.88 billion which is more than the HIT number.

\section{ACKNOWLEDGMENTS}

One of the authors (S. Y.) would like to thank G. Baskaran, B. Chakrabarti, N. V. Chalapathy, M. V. Ramdas, M. Tambe, and K. B. M. M. Krishna for valuable discussions.

1 World Health Organization, Coronavirus disease 2019 (COVID-19) situation report-10, (2020).

2 A. S. Fauci, H. C. Lane, R. R. Redfield, New England Journal of Medicine 382, 1268-1269 (2020). doi:10.1056/NEJMe2002387.

3 World Health Organization, Coronavirus disease 2019 (covid-19) situation report-51, (2020).

4 S. Flaxman, et al., DOI: https://doi.org/10.25561/77731.

5 C. Rothe, em et al., New England Journal of Medicine 382, 970-971 (2020). doi:10.1056/NEJMc2001468.

6 S. A. Lauer, et al., Annals of Internal Medicine (2020). URL: https://doi.org/10.7326/M200504 .

7 N. M. Linton, et al., J Clin Med. 2020;9. [PMID: 32079150] doi:10.3390/jcm9020538.

8 E. Dong, H. Du, and L. Gardner, The Lancet infectious diseases, DOI:https://doi.org/10.1016/S1473-3099(20)30120-1 (2020).

9 https://www.worldometers.info/coronavirus/

10 https://ourworldindata.org/grapher/total-covid-deaths-region

11 Eili Klein et al, CDDEP report (March 24, 2020) https://cddep.org/wpcontent/uploads/2020/03/covid19.indiasim.March23-2-eK.pdf

12 World Health Organization, World malaria report 2018 (see Fig. 1.1).

13 https://wwwnc.cdc.gov/travel/yellowbook/2020/travel-related-infectious-diseases/tuberculosis

14 Gregg N. Milligan and Alan D. T. Barrett, (2015). Vaccinology: an essential guide. Chichester, West Sussex: Wiley Blackwell. p. 310.

15 https://en.wikipedia.org/wiki/Timeline_of_the_2020_coronavirus_pandemic_in_India 